\title{
CYP2C9 and VKORC1 genotyping reagents from Idaho Technology: rapid turn-around, accurate results
}

Anticoagulation therapy is routinely used in settings ranging from the emergency room to presurgery. Idaho Technology has developed genotyping reagents targeting three loci: alleles * 2 and * 3 in the CYP2C9 gene and C.1 G-1639A in VKORC1. With a rapid turnaround time of $<1$ hour or the ability to increase throughput using traditional 96- or 384-well plate format, these reagents are appropriate for a range of settings.

Idaho Technology has developed rapid genotyping assays using SimpleProbe ${ }^{\mathrm{TM}}$ chemistry, based on fluorophore quenching ${ }^{1}$, to genotype the CYP2C9 ${ }^{*} 2$ and $* 3$ alleles as well as the VKORC1 G1639A single-nucleotide polymorphism. The assays are compatible with various Idaho Technology instrument platforms as well as several realtime PCR platforms from different manufacturers. The SimpleProbes are labeled with a common fluorescein isomer via proprietary linker chemistry and designed to perfectly match the sequence of the expected variant, thus creating a single-base mismatch when the wild-type sequence is present. This results in a low-temperature melting peak from the probe when the wild-type allele is present and a higher-temperature melting peak from the probe when the variant allele is present (Fig. 1). A single SimpleProbe detects both alleles, and, because the probe is a perfect match to the variant allele, false positives are virtually eliminated. Alternative, rare sequence variants have been identified in the CYP2C9 gene in close proximity to the *2 and *3 alleles, and these variants can produce false positives using other genotyping technologies. Owing to the design of the Idaho Technology probes, these rare variants, if present, introduce a second base-pairing mismatch under the probe. As expected, this results in even lower-temperature probe melting peaks than those observed from the single-mismatch wild-type allele-to-probe hybrid (Figs. 2 and 3).

\section{Applications of the technology}

Expectations for appropriate personalized or individualized medicine were high upon completion of the Human Genome Project. One of the most immediate applications is to reduce adverse drug reactions through dosage prediction based on a patient's personal genetic makeup. Despite several known gene-drug interactions and the demonstrated feasibility of pretherapy genotyping, the use of genetic testing to

\section{Jason McKinney, Ranae Lems \& Cameron Gundry}

Idaho Technology, Inc., Salt Lake City, Utah, USA. Correspondence should be addressed to J.M. (jasonm@idahotech.com) or C.G. (cameron_gundry@idahotech.com). guide dosage has not been widely incorporated into clinical practice. One reason for the lack of implementation of genotype-guided dosing has been the lack of a rapid, sensitive and robust genotyping method.

Warfarin (Coumadin) reduces heart attack and stroke risk and is the current mainstay of anticoagulation therapy. However, it has a narrow therapeutic range, and its use is complicated by wide interindividual
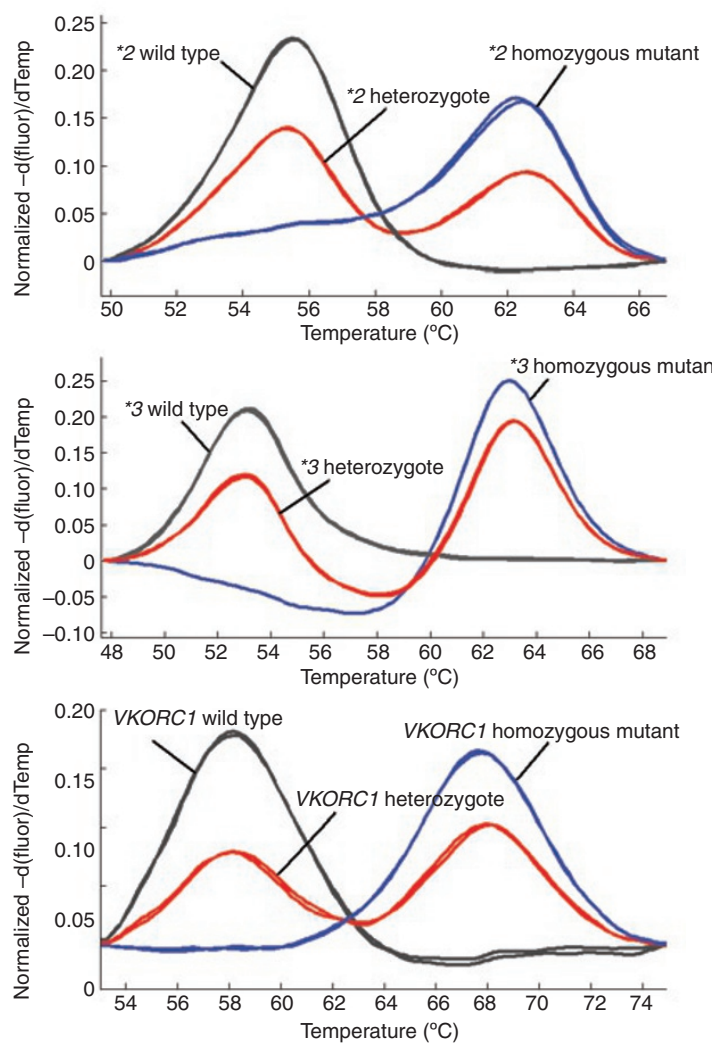

Figure 1 | Derivative normalized melting peaks representing all possible genotypes using Idaho Technology's warfarin genotyping reagents. 
APPLICATION NOTES

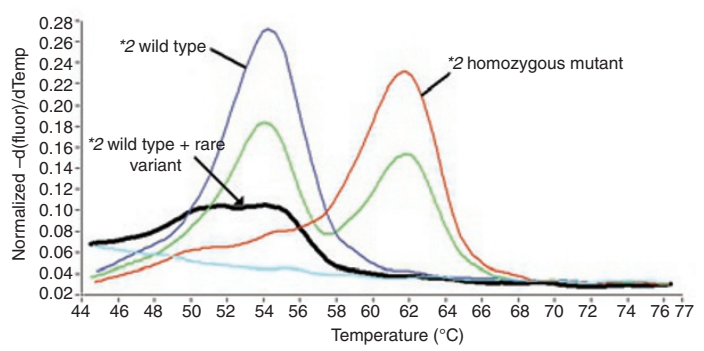

Figure 2 | Derivative melting peaks from Idaho Technology's CYP2C9*2 genotyping reagents. Genotypes are labeled. The rare variant causes further destabilization owing to a second mismatch under the probe in addition to the mismatch with the wild-type allele.

variability in dosing requirements. This variability leads to erratic initial dose responses, with increased bleeding risk associated with overanticoagulation or risk for a thrombo-embolic event if anticoagulation is subtherapeutic.

About $12 \%$ of dosage variability between patients has been found to depend on CYP2C9 polymorphisms. The CYP2C9*2 variant, a C to $T$ substitution at codon 144 (c.332), results in the replacement of arginine by cysteine and occurs in $11 \%-12 \%$ of the population. The CYP2C9*3 variant, an A to C substitution in codon 359 (c.813), results in replacement of isoleucine with leucine and is present in $7 \%-9 \%$ of the population. These coding variations produce reductions in enzymatic activity of $30 \%$ for *2-allele and $80 \%$ for *3-allele homozygotes. It was previously noted that individuals with one or more CYP2C9 variants had a protracted time to achieve a stable therapeutic range (international normalized ratio; INR) as well as increased risk for severe or life-threatening bleeding ${ }^{2,3}$.

The gene for vitamin $\mathrm{K}$ epoxide reductase complex subunit I (VKORC1) was recently identified along with what is suspected to be a functional variant in VKORC1 (c.1 G-1639A) associated with variable promoter activity. Polymorphisms in this gene account for about $30 \%$ of the dosage sensitivity variability 4,5 .

Genotyping for these single-nucleotide polymorphisms associated with altered warfarin dosage requirements is currently recommended by the US Food and Drug Administration (FDA) on the

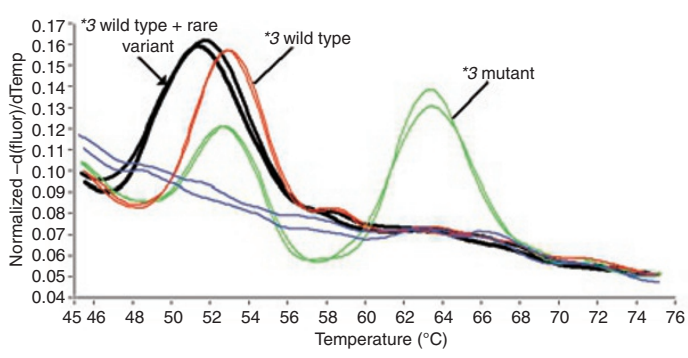

Figure 3 Derivative melting peaks from Idaho Technology's CYP2C9*3 genotyping reagents. Genotypes are labeled. The rare variant causes further destabilization owing to a second mismatch under the probe in addition to the mismatch with the wild-type allele.

medication labels, and commercially available reagents are available from several companies. Idaho Technology's advantage over other commercially available reagents is rapid turnaround time, low cost and the flexibility to use the assays on several platforms, some of which are already in place in several facilities. Coupled with a 10-minute DNA extraction from buccal swabs, time to result using Idaho Technology's assays is less than 1 hour, meaning initial warfarin dosing can be guided by genotype. To request information or a sample of our analyte-specific reagents for CYP2C9*2, CYP2C9*3 and VKORC1 c.1 G-1639A testing, visit our website (http://www. idahotech.com/) or contact us at Idaho Technology.

1. Crockett, A.O. \& Wittwer, C.T. Fluorescein-labeled oligonucleotides for real-time PCR: using the inherent quenching of deoxyguanosine nucleotides. Anal. Biochem. 290, 89-97 (2001)

2. Carlquist, J.F. et al. Rapid melting curve analysis for genetic variants that underlie inter-individual variability in stable warfarin dosing. J. Thromb. Thrombolysis $\mathbf{2 6}$ $1-7$ (2008)

3. Anderson, J.L. et al. Randomized trial of genotype-guided versus standard warfarin dosing in patients initiating oral anticoagulation. Circulation 116, 2563-2570 (2007).

4. Rieder, M.J. et al. Effect of VKORC1 haplotypes on transcriptional regulation and warfarin dose. N. Engl. J. Med. 352, 2285-2293 (2005).

5. Wadelius, M. et al. Association of warfarin dose with genes involved in its action and metabolism. Hum. Genet. 121, 23-34 (2007).

This article was submitted to Nature Methods by a commercial organization and has not been peer reviewed. Nature Methods takes no responsibility for the accuracy or otherwise of the information provided. 